\title{
Resistance to changes in diet
}

\author{
Richard Shepherd \\ Department of Psychology, University of Surrey, Guildford, Surrey GU2 7XH, UK
}

\begin{abstract}
Dietary changes can be difficult to effect both at an individual and at a population level, and even when changes do occur they are often far slower and less pronounced than might be expected. Three possible reasons for this situation will be considered: the complexity of food choice and competing influences, attitudinal ambivalence and optimistic bias. Food choice is influenced by a large number of factors, not only health considerations, and therefore it is not surprising that interventions based primarily on health concerns have been ineffective. Another concern is that people do not always have clear-cut attitudes, but rather can be ambivalent about foods and about healthy eating, and this factor might impact on the translation of beliefs and attitudes into behaviour. A third possible reason is optimistic bias, where individuals believe themselves to be at less risk from various hazards than is the average person. This effect has been demonstrated for nutritional risks, and this factor might lead people to take less note of health education messages. The stages-of-change model from health psychology has been proposed as a method for improving the effectiveness of behaviour change interventions. However, there are a number of problems in transferring such a model from smoking, where it was originally developed, to dietary behaviours, including the lack of clear-cut specific behaviours and behaviour change targets in the dietary field.
\end{abstract}

Attitudes: Dietary change: Optimistic bias: States of change

Influencing dietary choices in an effective way is not easy. Given recommendations, for example, to reduce fat in the diet (Department of Health, 1994) or to increase the consumption of fruit and vegetables, it is necessary to understand what determines people's choices of foods and what obstacles there might be to effecting such changes. Official recommendations have been in place in the UK since 1984 (Department of Health, 1984) for a reduction in the energy in the diet derived from fat, but the changes in fat as a percentage of energy at the population level have been slow.

There are a number of possible reasons for the lack of effectiveness of attempts at dietary change. Three specific issues will be discussed here: the complexity of human food choice and competing influences, attitudinal ambivalence and optimistic bias. This discussion will be followed by one of the stages-of-change model (Prochaska \& DiClemente, 1983) as a possible means for implementing dietary changes more effectively.

\section{Competing influences on food choice}

Food choice is a complex human behaviour, or set of behaviours, and therefore is influenced by many interrelating factors. It is not determined entirely by physiological or nutritional need, or by beliefs about and interest in the health benefits of particular foods or diets. Rather, it is influenced by a complex set of factors ranging from biological mechanisms and genetics on the one hand, to social and cultural factors on the other.

A number of models have been presented in the literature (for example, see Pilgrim, 1957; Khan, 1981; Randall \& Sanjur, 1981; Shepherd, 1985; Furst et al. 1996; Sobal et al. 1998; for review, see Shepherd, 1989) which aim to show the types of factors which might influence food choices. Such models are useful in pointing to the variables to consider in studies of food choice, but also emphasise the complexity of food choice behaviour. Fig. 1 shows an example of a model which schematically represents some of the factors affecting food choice. The factors influencing food choice are divided into those related to the food, to the person making the choice and to the external economic and social environment within which the choice is made.

Within the food itself there will be chemical components and physical properties which will have an impact on choice. One of the main routes through which this process will occur is sensory perception. Thus, some chemical and

Abbreviation: TPB, Theory of Planned Behaviour.

Corresponding author: Dr Richard Shepherd, fax + 44 1483 689553, email R.Shepherd@ surrey.ac.uk 


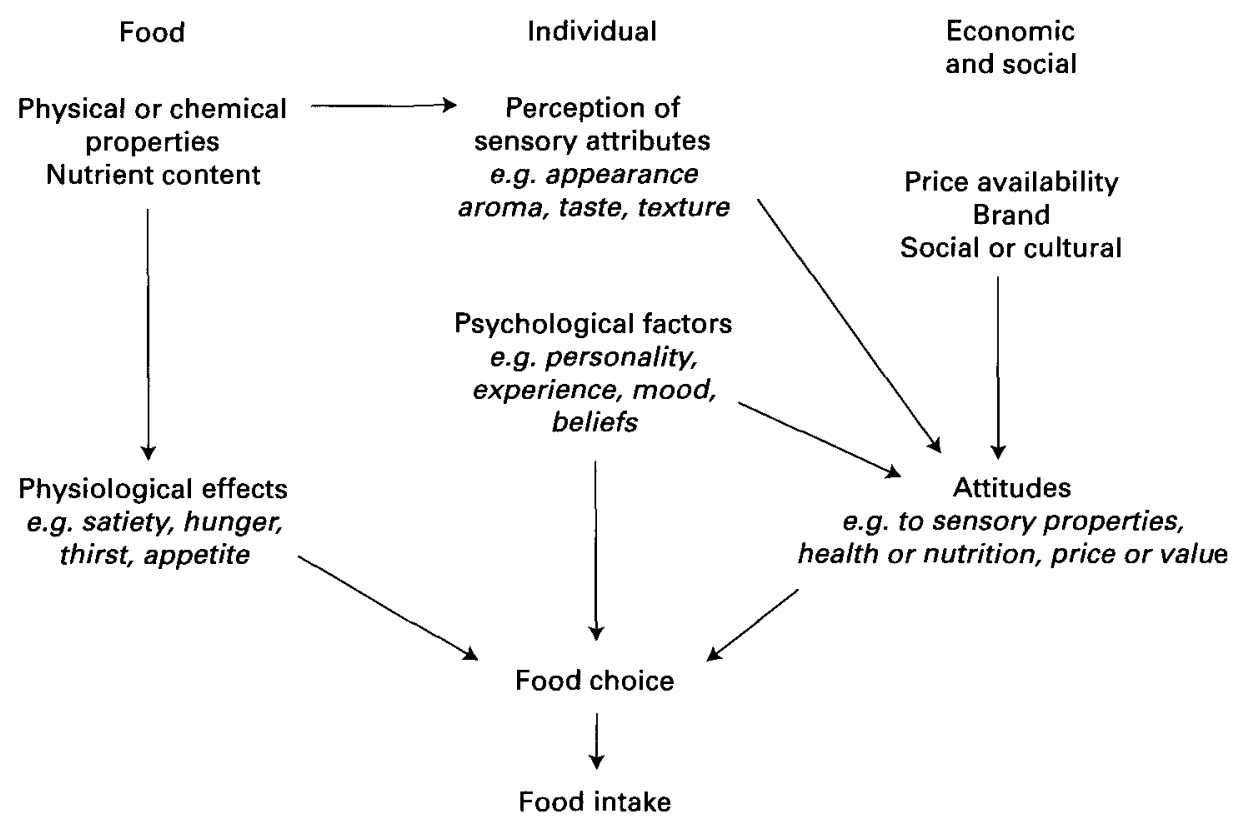

Fig. 1. A schematic representation of some factors affecting food choice and intake. (From Shepherd, 1985.)

physical properties of the food will be perceived by the person in terms of sensory attributes, e.g. flavour, texture or appearance. However, perceiving a particular sensory attribute in a particular food does not necessarily mean that the person will or will not choose to consume that food. Rather, it is the person's liking for that attribute in that food which will influence choice. Other chemical components in the foods, such as the amount of protein or carbohydrate, will have effects on the person, e.g. reducing hunger, and the learning of the association between the sensory attributes of a food and its post-ingestional consequences appears to be a major mechanism by which preferences develop (for example, see Hill \& Blundell, 1986). Psychological differences between people, such as personality, may also influence food choice; thus, for example, people with a higher non-discretionary salt intake show higher scores for extraversion $(P<0.05)$, but lower scores for neuroticism $(P<0 \cdot 01$; Shepherd \& Farleigh, 1986).

In addition to factors associated with the person and the food, there are also many other factors in the context within which the choice is made that can be very important in food choice. These factors include marketing and economic variables as well as social, cultural, religious or demographic factors (Murcott, 1989; Shepherd, 1989). The culture in which a person is brought up has a very strong influence on the types of choices made, and social interactions can have a profound effect on our views of foods and our eating behaviour. The impact of these factors has been receiving increasing attention (For example, see Murcott, 1998).

Fig. 1 shows a very simplified model of some of the likely influences on food choice. The impact of different factors is likely to vary across different types of choices, e.g. choosing a sandwich for lunch $v$. Christmas dinner or a celebration meal. Also, there is the complication that nutritionists are not usually interested in the choice of particular foods, but rather are interested in diets and the intakes either of types of foods (e.g. fruits) or in the outcome of the choice of many different types of foods, e.g. fat intake or $\mathrm{Ca}$ intake. It has long been recognised that there is a need to present nutritional and health information to consumers by referring to foods rather than to nutrients, but if the aim is to change, for example, fat intake there are many potential changes in behaviour which could achieve this aim, and the changes are likely to differ across the population. The translation of nutritional messages into specific behaviour changes that individuals need to make is far from easy, and this factor may be one of the reasons why dietary interventions are not very effective.

Given the earlier discussion it is not surprising that messages on health and nutrition have relatively little impact. There are many competing influences on people's choices of foods and their motives for particular choices are complex. Thus, an intervention which is primarily related to health may not have much impact.

\section{Attitudinal ambivalence}

One potential route towards behaviour change is through interventions seeking to change beliefs and attitudes (Eagly \& Chaiken, 1993). Within an attitude model such as the Theory of Planned Behaviour (TPB; Ajzen, 1988) behaviour is seen as following from intention, and in turn intention is a function of attitude, perceived social pressure and perceived control (Shepherd \& Raats, 1995). Attitude is predicted by the product of beliefs and evaluations of outcomes. Thus, an intervention based on such a model would target beliefs and attitudes as a possible route towards behaviour change.

The predominant view of attitudes in social psychology (and in the TPB) is that people hold positive or negative views concerning an attitude object, and that this attitude is then related to behaviour (Eagly \& Chaiken, 1993). 
According to this type of view people can be indifferent about a topic, but it does not allow for individuals holding simultaneously positive and negative feelings. However, people might be expected to be ambivalent about many topics and issues, and food is an issue where ambivalence might be expected to be particularly strong. In popular coverage of food issues, foods are often characterised as tasting nice and yet being unhealthy, and therefore it might be expected that people will have mixed feelings about consuming particular foods or about diet in general.

When people hold ambivalent attitudes or have mixed feelings it might be expected that attitudes would be less clearly related to behaviour. Also, attempts to change behaviour through changing beliefs and attitudes might be more difficult where attitudes are less well structured and/or there is a less clearly defined attitude-behaviour link.

The effect of ambivalence as a moderator of the relationship between attitudes and intention has been examined in several studies (for example, see Sparks et al. 1992). In one study (Sparks et al. 2001) we examined attitudes towards the consumption of chocolate and meat. The study was conducted within the framework of the TPB (Ajzen, 1988), which allows testing quantitatively the relationships between attitudes, intention and behaviour within a structured framework (Shepherd \& Raats, 1995). In addition to the standard components of the TPB, ambivalence was assessed by asking separate questions about positive and negative aspects of the behaviour. Thus, in one question, participants were asked to consider only the positive things about eating chocolate (or meat) and to ignore any negative things about it, and then to rate only those positive things from 'not at all positive' to 'extremely positive'. A similarly worded question assessed negative aspects on a scale from 'not at all negative' to 'extremely negative'. The ratings on these scales were then combined using the formula from Thompson et al. (1995).

In order to test whether the attitude-intention relationship was attenuated for those participants with higher ambivalence, a multiple regression was computed predicting intention from attitudes, ambivalence and the attitude $\times$ ambivalence product (with scores centred; Cohen $\&$ Cohen, 1983). We would predict a strong effect of attitude alone, based on previous results from the TPB. We would also predict a significant negative effect for the interaction between attitude and ambivalence, i.e. a negative $\beta$ coefficient in the multiple regression. This negative effect was found both for chocolate and for meat, as shown in Table 1. There was no effect for ambivalence alone, showing that (after taking account of attitude) higher levels of ambivalence do not themselves relate to higher or lower intention. Rather, the effect is for higher levels of ambivalence to attenuate the attitude-intention relationship.

Those participants who are more ambivalent and have more mixed feelings about consumption of foods tend to have a less clear relationship between attitudes and intention. Positive and negative views may be more or less salient in different contexts, and therefore more immediate sensory responses may predominate when confronted with foods, whereas the participant may focus on more healthrelated beliefs in the absence of food. The implications of
Table 1. Standardised regression coefficients $(\beta)$ from multiple regressions of intention to consume chocolate and meat on attitude, ambivalence and the product of attitude and ambivalence (adapted from Sparks et al. 2001)

\begin{tabular}{lcc} 
& \multicolumn{2}{c}{$\beta$} \\
\cline { 2 - 3 }$n \ldots$ & Chocolate & Meat \\
$R^{2} \ldots$ & 154 & 156 \\
Predictor & 0.35 & 0.54 \\
\hline Attitude & & \\
Ambivalence & $0.57^{\star \star \star}$ & $0.64^{\star \star \star}$ \\
Attitude $\times$ ambivalence & 0.01 & -0.06 \\
\hline
\end{tabular}

${ }^{\star} P<0.05,{ }^{\star \star} P<0.01,{ }^{* \star \star} P<0.001$.

$\dagger P<0.10$.

ambivalence for dietary behaviour, and in particular dietary change, need to be explored further.

\section{Optimistic bias}

One of the major problems in getting people to change their behaviour is the need to get them to recognise the need to change. This problem can be related to 'optimistic bias', which is a phenomenon where people underestimate the risk to themselves relative to others from a variety of hazards (for example, see Weinstein, 1987, 1989). It is also sometimes referred to as unrealistic optimism or over-optimism. Optimistic bias can be illustrated by asking a question such as 'Compared with other men/women my age, my chances of having a heart attack in the future are ...' with responses on a scale running from 'much below average' to 'much above average', and a mid-point of 'average for men/women my age'. Weinstein (1989) demonstrated that, at the group level, there is a consistent effect of participants marking their own personal risk as below average. However, if the sample of participants is representative of the appropriate population (i.e. they are not a special group such as nutritionists comparing themselves to the general population) then the mean response over the sample of participants should be near the centre of the response scale, i.e. average risk.

An alternative method for assessing optimistic bias is to ask for separate ratings of personal risk and risk for 'other people'. Frewer et al. (1994) had 'people' rate risks from a number of different types of hazards associated with foods. The potential hazards included a high-fat diet, along with microbiological and technological risks (e.g. genetically modified foods). In each case participants rated the risk to themselves and separately rated the risk to 'other people'. Optimistic bias was found for all the hazards included in the study. Participants saw themselves as at significantly less risk than other people for all the hazards included (all $P<0 \cdot 001$ ). However, these effects were larger for some of the hazards, being particularly marked for the lifestyle hazards of a high-fat diet and alcohol abuse, as well as for food poisoning from home-prepared foods.

In the same study participants were also asked to rate the degree of control they felt that they had over the same set of hazards, again asking separate questions for personal control and control by the average person. Participants saw themselves as having more control than other people over 
lifestyle hazards (e.g. high-fat diet and alcohol abuse), and the absolute levels of ratings of control were very high for these hazards. This finding confirms results from other studies that feelings of control are related to increased optimistic bias, and it would appear that participants see nutritional risks both as ones over which they have personal control, and where they are at less risk than other similar people.

Sparks et al. (1995) asked participants to rate the chances of their putting on weight, having heart disease and being unwell because of a high-fat diet. In this case the ratings were direct comparisons of these outcomes relative to other people of the same age and gender, on a scale ranging from 'much below average' through 'average' to 'much above average'. In each case they rated their susceptibility as less than average. These same participants also rated their consumption of cheese, meat, fat, margarine and/or butter, and biscuits, buns, cakes and pastries relative to the average person of the same age and gender. In each case, except cheese, participants rated their consumption of these foods as significantly less than the average $(P<0.001)$.

These results show that people have a positive view of the risks to themselves from various hazards and also have a positive view of their own dietary behaviour, both in terms of intake of particular nutrients and of specific foods. There is still, of course, a question as to whether this view has any impact on their behaviour.

Paisley \& Sparks (1998) used the TPB to investigate whether 'perceived need' to reduce fat intake had an impact on intention to change. Perceived need was assessed by asking participants 'Do you feel that you need to reduce your fat intake' with responses on a five-point scale labelled 'no, need to increase', 'not at all', 'slightly', 'a great deal' and 'a very great deal'. A regression showed a significant $(P<0.01)$ increase in variance accounted for with the addition of perceived need after the initial inclusion of the components of the TPB. Thus, perceived need is a significant $(P<0.01)$ predictor of intentions to reduce fat intake even when other important determining factors are taken into account. The feeling of the need to change therefore has an impact, and if people feel that their diet is already healthy and that they are at less risk than other people then they may be less likely to implement change.

Optimistic bias is clearly of importance if we are interested in how people think about risks and how their views on risks influence their behaviour. A number of explanations have been put forward for such biases. There may be a need to deny risks in order to avoid anxiety, or people may not consider the likely actions taken by other people to avoid risks, thereby attaching too much weight to their own riskavoiding behaviours (Weinstein, 1984). Whatever the cause of such biases, it is extremely difficult to reduce them (Weinstein \& Klein, 1995), and therefore attempts to change bias may not be fruitful as a means of making dietary interventions more effective. However, it needs to be taken into account in attempts to change dietary behaviour.

\section{Stages-of-change model}

Optimistic bias points to one of the main issues in dietary intervention: the need to tailor information more for individuals rather than simply presenting general messages to everyone (Brug et al. 1997). This issue is also highlighted in the transtheoretical model (sometimes referred to as the stages-of-change model) developed by Prochaska \& DiClemente (1983) and Prochaska et al. (1992). Originally this model was developed from an analysis of different types of therapy for changing behaviours, and in particular addictive behaviours such as smoking. Most applications are still on addictive behaviours, but the model seeks to be applicable to other forms of behaviour change, and there have been more recent applications to other forms of behaviour, including exercise (Courneya et al. 2001) and diet (Greene et al. 1994; Horwath, 1999).

There are a number of important aspects of the transtheoretical model. The first is the idea that when participants change a behaviour they go through a series of stages rather than it being a continuous process; these stages are precontemplation, contemplation, preparation, action and maintenance. Someone in precontemplation does not recognise the need to change and, therefore, has no intention to change. Someone in contemplation recognises that a problem exists and is thinking about changing, but has not yet made definite commitments to change. Preparation is defined by Prochaska et al. (1992) as comprising those participants who are intending to take action within the next month and have unsuccessfully taken action within the last year. In the action stage major changes are made to the behaviour, and this stage lasts from the first day until 6 months after such changes. The maintenance stage is when the changes have persisted for more than 6 months. Relapse is also sometimes included as a stage, since in many cases (and perhaps the majority for some behaviours) participants will move back rather than simply progressing through each of the stages in turn.

In addition to the idea of stages of change, the transtheoretical model also introduces the idea that different processes will be matched to the various stages (Prochaska \& DiClemente, 1983). Thus, in the early stages consciousnessraising and other cognitive strategies will be important, whereas in the later stages (action and maintenance) behavioural processes will become more important.

This model has been applied extensively to addictive behaviours, and in particular smoking, with some degree of success (Prochaska \& DiClemente, 1983; Prochaska et al. 1992). However, it is presented as a model for all forms of behaviour change, and therefore should be applicable to effecting changes in dietary behaviours. There have been a number applications of this type of model within the dietary area, including reducing fat intake, increasing fruit and vegetable consumption, and weight loss or weight control (for review, see Horwath, 1999).

While the applications to addictive behaviours have shown some success, the model is not without its critics (for example, see Sutton, 1996), and its application to dietary behaviours is far more problematic. Weinstein et al. (1998) point to a number of criteria that should be met in order to have a useful stage model. These criteria include the need to have an accurate method for classifying participants into the appropriate stage. Second, most participants should move through the stages in the specified sequence, although there may be some exceptions to this process. Participants at the 
same stage should face similar issues, and therefore the model allows these issues to be addressed for a group of participants identified as being at the same stage. Finally, different factors should be important for different stage transitions. If all stage transitions are dependent on the same factors, then there is no advantage in having a staged model, and the same intervention will be effective no matter what stage a participant is at. The advantage of a stage model should be that interventions can be targeted specifically at participants seeking to move from one stage to the next and that intervention would not be effective for someone at a different stage.

The boundaries between stages are not hard and fast, and in particular the stage timing appears to be arbitrary (Povey et al. 1999); after 6 months a participant moves from action to maintenance simply by virtue of the time spent acting in a particular way, but this interval could be set at 1 month, 3 months or any other time interval. Also, given the definition of the preparation stage presented earlier, with the participant having some previous unsuccessful attempts at behaviour change, it is not clear how someone can be in the preparation stage on their first time through the stages (Weinstein et al. 1998). Movement through the stages does not follow the correct pattern in many cases and, while some exceptions would be expected, if a great many participants do not follow the standard pattern, the usefulness of a stage model is called into question. Processes may occur at more than one stage, and therefore the differentiation between stages is not really clear-cut, and even when processes can be shown to be used at a particular stage, it might be because they are inhibiting movement to the next stage rather than being the most useful processes at that stage.

The earlier criticisms relate to the application of the stages-of-change model to any behaviour change. Application of the stages-of-change model to dietary behaviours brings with it an extra set of problems in conceptualisation and application of the model. The first is that the goal in dietary changes is not elimination, as for addictions, but rather some level of intake or consumption (e.g. $35 \%$ food energy from fat, five portions of fruit and vegetables daily). The second is that in many cases these targets are in the form of an outcome rather than a behaviour. Hence, consuming $35 \%$ energy from fat is not a behaviour, but rather a participant eats a set of foods and this nutrient intake is the outcome of that set of behaviours. A further consequence of this situation is that participants do not know when they have reached the target, since they do not know their own nutrient intake, either before they have changed or after. Although this problem is eased with other types of behaviour, such as fruit and vegetable consumption, where participants can more easily estimate whether they do consume five portions daily, the situation is not as straightforward as smoking where the goal is not to smoke at all. In many dietary studies there are no clear end targets set; the aim might be 'to increase fruit and vegetable consumption', 'to reduce fat intake' or 'to avoid high-fat foods'. In these cases it is difficult, both for the participant and the researcher, to know whether the target has been achieved, and in many cases it would be possible to change further. With dietary behaviour there are multiple changes needed; for example, changing the amounts consumed of several foods rather than changing a single behaviour (as in smoking). Although it could be argued that emphasis should be given to a clear and definable behaviour, since that is how the model is conceptualised, there are problems with this approach, since often practitioners are interested in outcomes (e.g. fat intake) rather than a specific behaviour (e.g. eating chocolate biscuits).

One of the consequences of these differences between dietary behaviours and smoking is that where targets are specified (e.g. $35 \%$ energy from fat) a number of participants who classify themselves as maintainers fail to meet the target. There are problems classifying these participants into the same group as those who have achieved the target and are maintaining it. This situation led Greene et al. (1994) to reclassify these participants into the preparation stage, while others have suggested putting them into the precontemplation stage, since they are not considering further change (Brug et al. 1997). However, neither of these strategies overcomes the problem, since psychologically these 'pseudo-maintainers' are not similar to those in preparation or in precontemplation, and it would not be expected that interventions designed for participants in those stages would be effective with them. Alternatives might be to reclassify them into a new group of 'failed maintainers' and to develop interventions specifically for that group, or to take into account the idea that dietary change is a series of changes and assess whether participants placing themselves in maintenance are interested in further change.

Horwath (1999) reviews thirty-four dietary studies which take the transtheoretical model as a starting point. However, the majority of these studies are cross-sectional, simply classifying participants into stages and then relating one or more of the transtheoretical model constructs (e.g. processes, decisional balance, self-efficacy) to the stage. In order to test whether the model is really effective it is necessary to carry out interventions, testing stage-matched interventions $v$. controls of mismatched interventions or general interventions, in order to demonstrate that the model offers some advantage over other forms of intervention. Horwath (1999) lists only one study which meets this criterion. Thus, although this model has some potential for more effectively tailoring information for participants seeking to change their diets, it requires more critical testing in this field.

\section{Conclusions}

Dietary change is difficult to implement effectively. There are many potential reasons for this problem. One of the most important is likely to be the competing influences on food choice which will reduce the effectiveness of interventions based entirely on the health benefits of specific dietary changes. Food choice is complex and is influenced by a large range of disparate factors. Interventions must take account of these competing influences and take note of the likely mechanisms underlying food preferences and food choice. There are other more specific factors which might impede the effectiveness of interventions, including ambivalence and optimistic bias. Overcoming optimistic bias is not easy, but messages which are more personalised may offer one means for addressing some of these issues. 
The transtheoretical, or stages-of-change, model appears to offer one means for improving behaviour change through allowing more tailored and personalised interventions. However, due note needs to be taken of the differences between the addictive behaviours, such as smoking, for which this model was originally developed, and the types of behaviours of interest to nutritionists. This difference might necessitate the development of different constructs or different measures of existing constructs specifically for dietary behaviours. While the model offers a means for classifying participants and targeting messages more effectively, it needs to be tested critically in order to determine whether it really is a useful addition to dietary intervention strategies.

\section{References}

Ajzen I (1988) Attitudes, Personality, and Behavior. Milton Keynes, Bucks.: Open University Press.

Brug J, Glanz K \& Kok G (1997) The relationship between selfefficacy, attitudes, intake compared to others, consumption, and stages of change related to fruit and vegetables. American Journal of Health Promotion 12, 25-30.

Cohen J \& Cohen P (1983) Applied Multiple Regression/ Correlation Analysis for the Behavioral Sciences. Hillsdale, NJ: Lawrence Erlbaum.

Department of Health (1984) Diet and Cardiovascular Disease. Report on Health and Social Subjects no. 28. London: H.M. Stationery Office.

Department of Health (1994) Nutritional Aspects of Cardiovascular Disease. Report on Health and Social Subjects no. 46. London: H.M. Stationery Office.

Courneya KS, Plotnikoff RC, Hotz SB \& Birkett NJ (2001) Predicting exercise stage transitions over two consecutive 6-month periods: a test of the theory of planned behaviour in a population-based sample. British Journal of Health Psychology 6, 135-150.

Eagly AH \& Chaiken S (1993) The Psychology of Attitudes. San Diego, CA: Harcourt, Brace and Jovanovich.

Frewer LJ, Shepherd R \& Sparks P (1994) The interrelationship between perceived knowledge, control and risk associated with a range of food related hazards targeted at the self, other people and society. Journal of Food Safety 14, 19-40.

Furst T, Connors M, Bisgoni CA, Sobal J \& Falk LW (1996) Food choice: a conceptual model of the process. Appetite $\mathbf{2 6}$, 247-266.

Greene GW, Rossi SR, Reed GR, Willey C \& Prochaska JO (1994) Stages of change for reducing dietary fat to $30 \%$ of energy or less. Journal of the American Dietetic Association 94, 1105-1110.

Hill AJ \& Blundell JE (1986) Macronutrients and satiety: the effects of a high-protein or high-carbohydrate meal on subjective motivation to eat and food preferences. Nutrition and Behavior 3, 133-144.

Horwath CC (1999) Applying the transtheoretical model to eating behaviour change: challenges and opportunities. Nutrition Research Reviews 12, 281-317.

Khan MA (1981) Evaluation of food selection patterns and preferences. CRC Critical Reviews in Food Science and Nutrition 15, 129-153.

Murcott A (1989) Sociological and social anthropological approaches to food and eating. World Review of Nutrition and Dietetics 55, 1-40.

Murcott A (editor) (1998) The Nation's Diet: The Social Science of Food Choice. Harlow, Essex: Adison Wesley.
Paisley CM \& Sparks P (1998) Expectations of reducing fat intake: the role of perceived need within the Theory of Planned Behaviour. Psychology and Health 13, 341-353.

Pilgrim FJ (1957) The components of food acceptance and their measurement. American Journal of Clinical Nutrition 5, 171-175.

Povey R, Conner M, Sparks P, James R \& Shepherd R (1999) A critical examination of the application of the Transtheoretical Model's stages of change to dietary behaviours. Health Education Research 14, 641-651.

Prochaska JO \& DiClemente CC (1983) Stages and processes of self-change of smoking - toward an integrative model of change. Journal of Consulting and Clinical Psychology 51, 390-395.

Prochaska JO, DiClemente CC \& Norcross JC (1992) In search of how people change - applications to addictive behaviors. American Psychologist 47, 1102-1114.

Randall E \& Sanjur D (1981) Food preferences - their conceptualization and relationship to consumption. Ecology of Food and Nutrition 11, 151-161.

Shepherd R (1985) Dietary salt intake. Nutrition and Food Science 96, 10-11.

Shepherd R (editor) (1989) Factors influencing food preferences and choice. In Handbook of the Psychophysiology of Human Eating, pp. 3-24. Chichester, West Sussex: Wiley.

Shepherd R \& Farleigh CA (1986) Preferences, attitudes and personality as determinants of salt intake. Human Nutrition: Applied Nutrition 40A, 195-208.

Shepherd R \& Raats MM (1995) Attitudes and beliefs in food habits. In Food Choice, Acceptance and Consumption, pp. 346-364 [HL Meiselman and HJH MacFie, editors]. London: Blackie Academic and Professional.

Sobal J, Khan LK \& Bisogni C (1998) A conceptual model of the food and nutrition system. Social Science and Medicine 47, 853-863.

Sparks P, Hedderley D \& Shepherd R (1992) An investigation into the relationship between perceived control, attitude variability and the consumption of two common foods. European Journal of Social Psychology 22, 55-71.

Sparks P, James R, Conner M, Shepherd R \& Povey R (2001) Ambivalence about health-related behaviors: an exploration in the domain of food choice. British Journal of Health Psychology 6, 53-68.

Sparks P, Shepherd R, Wieringa N \& Zimmermanns N (1995) Perceived behavioural control, unrealistic optimism and dietary change: an exploratory study. Appetite 24, 243-255.

Sutton S (1996) Can 'stages of change' provide guidance in the treatment of addictions? A critical examination of Prochaska and DiClemente's model. In Psychotherapy, Psychological Treatments and Addictions, pp. 189-205 [G Edwards, editor]. Cambridge: Cambridge University Press.

Thompson M, Zanna M \& Griffin D (1995) Let's not be indifferent about (attitudinal) ambivalence. In Attitude Strength: Antecedents and Consequences, pp. 361-386 [RE Petty and JA Krosnick, editors]. Hillsdale, NJ: Lawrence Erlbaum.

Weinstein ND (1984) Why it won't happen to me: perceptions of risk factors and susceptibility. Health Psychology 3, 431-457.

Weinstein ND (1987) Unrealistic optimism about susceptibility to health problems: conclusions from a community-wide sample. Journal of Behavioral Medicine 10, 481-500.

Weinstein ND (1989) Optimistic biases about personal risks. Science 246, 1232-1233.

Weinstein ND \& Klein WM (1995) Resistance of personal risk perceptions to debiasing interventions. Health Psychology 14, $132-140$.

Weinstein ND, Rothman AJ \& Sutton SR (1998) Stage theories of health behavior: conceptual and methodological issues. Health Psychology 17, 290-299. 